\title{
Effects of Low Frequency Continuous Muscle Vibration on Learning and Transfer of a Knee Joint Positioning Task
}

\author{
Chelette, A.M. \\ Health \& Human Performance, Center for Neuromotor and \\ Biomechanics Research, University of Houston; National Center \\ for Human Performance, Texas Medical Center
}

\author{
Layne, C. S* \\ Health \& Human Performance, Center for Neuromotor and \\ Biomechanics Research, Center for Neuro-Engineering and \\ Cognitive Science, University of Houston; National Center for \\ Human Performance, Texas Medical Center
}

\begin{abstract}
The use of continuous low frequency vibration has been shown to improve perception of limb motion under certain conditions. The purpose of this study was to determine whether practice combined with continuous low frequency vibration could be used to enhance learning and subsequent transfer of a knee joint positioning task. Absolute and constant error were compared between a Control group, that did not receive vibration during practice and an Experimental group, that received $15 \mathrm{~Hz}$ vibration on the quadriceps tendon during each practice trial. Both groups were able to reduce absolute and constant error with practice but vibration had no impact on learning or transfer. These findings indicate that subjects effectively ignore the vibration induced input instead of using it in a beneficial way to augment the sensory input associated with limb motion. This suggests that the vibration induced haptic input may be down weighted by the sensory motor system such that ongoing movement was not disrupted. It is possible that vibration may only be useful when the haptic input generated by vibration is meaningfully related to the task.
\end{abstract}

Keywords: Vibration, proprioception, haptic devices.

Index Terms: H.5.2 [Information Interfaces and Presentation]: User interfaces --- Haptic I/O

\section{INTRODUCTION}

Typically, illusions of movement are induced in response to musculo-tendon vibration [1]. However, different frequencies of vibration selectively activate different receptors [2]. Type II receptors preferentially respond to low frequency vibration (10-20 $\mathrm{Hz}$ ) where as high frequency vibration typically induces a response in Type Ia receptors [2]. The differential mechanoreceptor responses to distinct frequency ranges allude to the possibility that the perceptual effects of different vibration frequencies may also be different.

Although Cordo et al [3] reported increased errors in positioning tasks with the use of vibration, an examination of the reported results indicate that lower frequency vibration, particularly when paired with specific movement velocities, is not as disruptive to perception of limb motion as higher frequency vibration. Moreover, a recent study reported that low frequency continuous vibration was actually beneficial for reducing error

*Corresponding author: clayne2@central.uh.edu

IEEE Haptics Symposium 2014

23-26 February, Houston, Tx, USA

U.S. Government work not protected by U.S. copyright and variability of elbow joint positioning [4]. However, in this study, vibration was applied with the use of a hand held dumbbell instead of directly to the musculo-tendons of the elbow joint [4]. Thus, it remains an open question as to the extent to which vibration applied directly to the musculo-tendons modifies the perceptual processes associated with limb motion.

If low frequency continuous vibration does improve perceptual functioning, it could potentially be beneficial for the training of persons with transtibial amputations. It is possible that enhanced perceptual learning that may occur with the use of vibration during limb movement could result in positive transfer to the affected limb of these patients. Improved perceptual awareness around the knee joint of the affected limb should result in enhanced control of the prosthesis. Currently, vibration is being explored as a tool to provide augmented feedback about limb force and motion to persons with upper limb amputations [6,7].

The purpose of this study was to determine if continuous low frequency vibration applied directly to musculo-tendons of the anterior thigh has benefits for the learning and bilateral transfer of a proprioceptive task involving the knee joint.

$\mathrm{H}_{1}$ : Overall there will be a significant reduction in practice leg error from Pre to Post practice.

$\mathrm{H}_{2}$ : Practice with vibration will have result in a greater reduction in practice leg error from Pre to Post practice.

$\mathrm{H}_{3}$ : Practice with vibration will result in more positive bilateral transfer (reduction in non-practice leg error) from Pre to Post practice.

\section{Methods}

Thirty healthy adults age $26 \pm 5$ years $(16 \mathrm{M}, 14 \mathrm{~F})$ participated in this study. Subjects provided informed consent before being randomly assigned to one of two groups: Control group (9 male, 6 female) and Experimental group (7 male, 8 female).

\subsection{Procedures}

Subjects were blindfolded and seated in a Biodex System 3 with their leg fastened to a dynamometer such that the knee was flexed at a $90^{\circ}$ angle. Subjects completed a novel task in which they were instructed to actively move their knee to the target position of $55^{\circ}$ on every trial. No resistance was applied during the movements. Subjects began the trial upon receiving a verbal "GO" signal and extended their knee until they believed they had reached the target angle. When they believed they had reached the target position, they used a remote stop button to lock their limb in place so that the joint position could be recorded. Before beginning the experiment, each leg was passively extended to $55^{\circ}$ and held briefly in place once to familiarize the subject with the target angle.

A Pre Test - Post Test design was used in which the task was performed with each leg three times before (Pre) and after (Post) a 
Table 1: Schedule of Experimental Trials for the Experimental and Control groups in which vibration was either present or absent.

\begin{tabular}{|c|c|c|c|c|}
\hline Leg & Experimental & Control & $\begin{array}{l}\text { Feedback } \\
\text { Frequency }\end{array}$ & Trials \\
\hline \multicolumn{5}{|c|}{ Pre Practice } \\
\hline $\begin{array}{l}\text { Left } \\
\text { Knee }\end{array}$ & Absent & Absent & None & $x 3$ \\
\hline $\begin{array}{l}\text { Right } \\
\text { Knee }\end{array}$ & Absent & Absent & None & $x 3$ \\
\hline \multicolumn{5}{|c|}{ Practice } \\
\hline $\begin{array}{l}\text { Right } \\
\text { Knee }\end{array}$ & Present & Absent & Every Trial & $\mathrm{x} 10$ \\
\hline $\begin{array}{l}\text { Right } \\
\text { Knee }\end{array}$ & Present & Absent & $\begin{array}{l}\text { Every 2nd } \\
\text { Trial }\end{array}$ & $\mathrm{x} 10$ \\
\hline $\begin{array}{l}\text { Right } \\
\text { Knee }\end{array}$ & Present & Absent & $\begin{array}{l}\text { Every 3rd } \\
\text { Trial }\end{array}$ & $\mathrm{x} 10$ \\
\hline $\begin{array}{l}\text { Right } \\
\text { Knee }\end{array}$ & Present & Absent & $\begin{array}{l}\text { Every 3rd } \\
\text { Trial }\end{array}$ & $\mathrm{x} 10$ \\
\hline $\begin{array}{l}\text { Right } \\
\text { Knee }\end{array}$ & Present & Absent & $\begin{array}{l}\text { Every 4th } \\
\text { Trial }\end{array}$ & $\mathrm{x} 10$ \\
\hline \multicolumn{5}{|c|}{ Post Practice } \\
\hline $\begin{array}{l}\text { Right } \\
\text { Knee }\end{array}$ & Absent & Absent & None & $x 3$ \\
\hline $\begin{array}{l}\text { Left } \\
\text { Knee }\end{array}$ & Absent & Absent & None & $x 3$ \\
\hline
\end{tabular}

practice period. There was a 5-minute break between the practice period and the Post practice Trials. The practice period consisted of 5 blocks of 10 trials in which only the right knee was used to perform the task. For reference see Table 1.

During practice subjects were provided verbal feedback about their knee joint angle immediately following their return to the starting position. For example, if their final knee position was $60^{\circ}$ for that trial they were told simply "sixty degrees". Feedback was provided on a faded frequency schedule so that feedback was provided after every trial of the first block, every second trial of the second block, and so on. During both the third and fourth block subjects received verbal feedback every third trial, but during the third block, they received feedback after 4 trials (trials $1,4,7,10)$ and during the $4^{\text {th }}$ block they received feedback after only 3 trials (trial 2, 5,8). Fading the frequency of feedback over the course of practice has been shown to be more effective for retention of performance gains than delivery of feedback after every trial [7]. The same type and frequency of verbal feedback was delivered to both groups.

During practice the Experimental group received vibration on every trial. A Vibrasens (C) Vb115 vibrator (Techno Concept, France) was placed on the quadriceps tendon of the participating leg and secured with an elastic strap. Vibration was applied continuously at a frequency of $15 \mathrm{~Hz}$ and 1-mm amplitude. Vibration was initiated immediately preceding the "Go" signal and ceased after the subject pressed the remote stop. The Control group did not receive vibration during practice.

\subsection{Analysis}

Absolute error was calculated as the absolute difference between the target position and the subject's actual recorded position. Constant error was calculated as the target value (55) minus the score retaining the direction of error (i.e. under or overshooting the target value). A transfer index (TI) was calculated as a measure of the magnitude of bilateral transfer of learning between limbs [8].

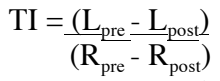

For absolute error TI, $\mathrm{L}_{\text {pre }}$ represents the mean of the absolute error of the left leg Pre practice trials, $\mathrm{L}_{\text {post }}$ the mean of the absolute error of the left leg Post practice trials, $R_{\text {pre }}$ the mean of the absolute error of the right leg Pre practice trials, and $R_{\text {post }}$ the

mean of the absolute error of the right leg Post practice trials. For constant error TI the formula was calculated the same way but using mean of the constant error for each leg Pre and Post. The equation was adapted to represent the expectation that Pre practice error will be larger than Post practice error, causing positive values greater than 1 to be indicative of greater positive bilateral transfer.

Repeated measures ANOVA was used to determine differences between Pre and Post test scores with time and leg as within group factors and group as the between group factor. Mann-Whitney U test was used to determine whether differences exist in either the absolute error or constant error Transfer Indices between the Experimental and Control groups.

\section{Results}

Changes in absolute error from Pre to Post practice trials in each group and leg are displayed in Figure 1. Overall, there was a significant main effect of time on absolute error (Pre vs. Post) $\left(\mathrm{F}_{(1,28)}=5.31 ; \mathrm{p}<.05 ; \mathrm{n}_{\mathrm{p}}^{2}=.159\right)$. Absolute error decreased from Pre practice $(\mathrm{M}=5.71, \mathrm{SD}=2.96)$ to Post practice $(\mathrm{M}=4.22, \mathrm{SD}$ $=2.94$ ) independent of group. Mean absolute error of the right leg reduced from 4.07 to 3.33 in the Experimental group, and reduced from 4.86 to 3.11 in the Control group. For the left leg, mean absolute error reduced from 6.0 to 4.80 in the Experimental group, and from 7.91 to 5.62 in the Control group. There was a significant effect of leg (left or right) $\left(\mathrm{F}_{(1,28)}=12.22 ; \mathrm{p}<.01 ; \mathrm{n}_{\mathrm{p}}{ }^{2}=\right.$ .30). Overall, the right leg had a smaller mean error $(\mathrm{M}=3.85$, $\mathrm{SD}=3.68)$ compared to the left $(\mathrm{M}=6.08, \mathrm{SD}=1.91)$. There were no interaction effects of leg with time or group (p>.05) on absolute error. There was not a main effect of group or an interaction effect of time on group ( $\mathrm{p}>.05$ ) on absolute error.

Changes in constant error from Pre to Post practice in each group and leg are displayed in Figure 2. There was a significant interaction effect of time with leg on constant error $\left(\mathrm{F}_{(1,28)}=6.15\right.$; $\mathrm{p}<.05 ; \mathrm{n}_{\mathrm{p}}^{2}=.18$ ). The difference in constant error between legs decreased from Pre to Post practice regardless of group. Pre practice mean constant error was 2.53 for left leg and -.733 for Right Leg. Post practice mean constant error was 2.789 for left leg and 2.756 for right leg. The mean of left leg constant error across groups stayed relatively the same while the mean of right leg constant error reversed directions and increased in magnitude. There were no main effects of time, leg, or group and no other interaction effects on constant error.

The mean TI of absolute error was $2.51(\mathrm{SD}=4.20)$ for the Experimental group and $2.23(\mathrm{SD}=5.22)$ for the Control group. The mean TI of constant error was $2.04(\mathrm{SD}=3.64)$ for the Experimental group and $.96(\mathrm{SD}=6.73)$ for the Control group. There was not a significant difference between groups on either the absolute error or constant error TI $(\mathrm{p}>.05)$.

\section{Discussion}

The purpose of this study was to determine if vibration was beneficial for the learning and bilateral transfer of a knee joint positioning task after practice. We hypothesized that after practice both groups would reduce their right leg error but that a greater reduction in error would be observed in the Experimental (i.e. vibration) group. In addition, we hypothesized that practice on the right leg would result in bilateral transfer of learning to the left leg and that a greater reduction in left leg errors would be observed in the Experimental group. 


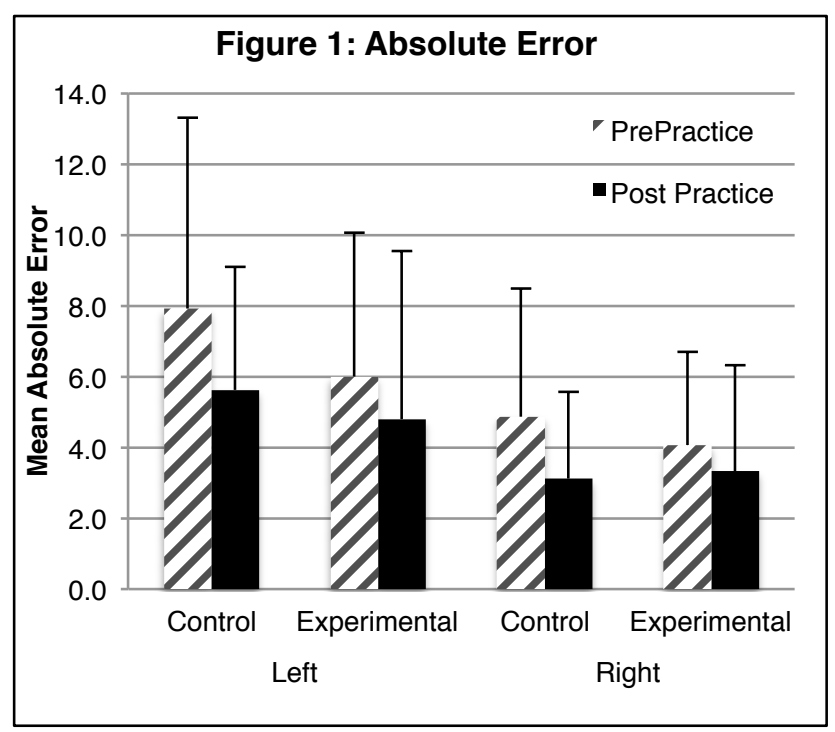

Figure 1: Mean absolute error of the right and left legs: Pre (stripes) and Post (solid) practice for both the Control and Experimental groups. Error Bars represent standard deviation.

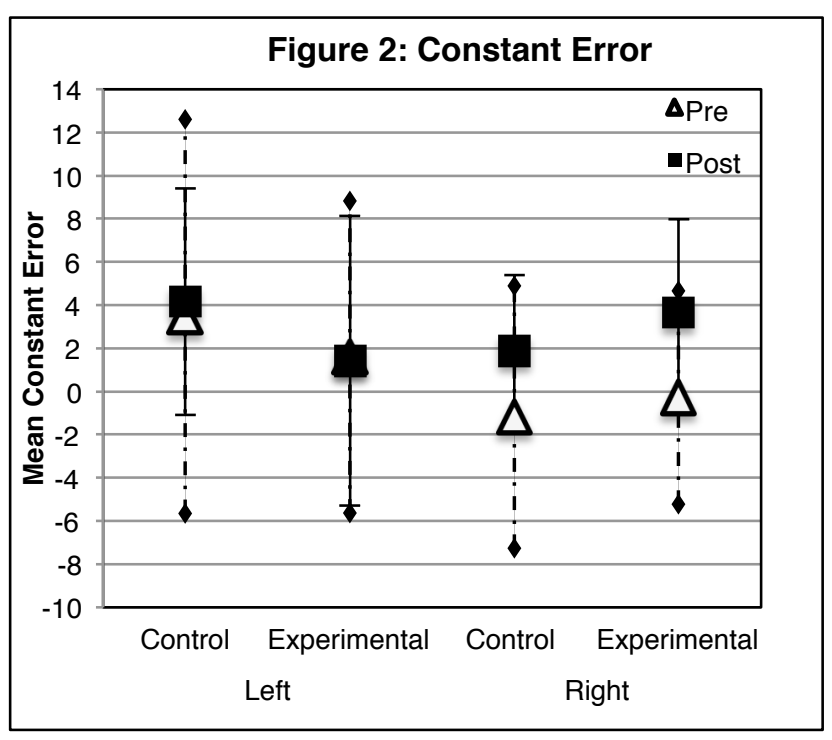

Figure 2: Mean constant error of the right and left legs: Pre (Open Triangle) and Post (Solid Square) practice for both the Control and Experimental groups. Error Bars represent standard deviation for Pre (dashed) and Post (solid) practice.

\subsection{Effect of practice}

Overall subjects significantly reduced absolute error with practice, however, constant error was not significantly changed by practice. Although the mean of right leg constant error reversed directions and increased in magnitude after practice, scores varied greatly in both directions about the target. Since absolute error averages are not affected by variability of direction, it is a better representation of the change in magnitude of error with practice. So our first hypotheses that the magnitude of error would reduce with practice regardless of group is supported. This is not surprising, as traditionally practice invariably results in performance improvements.

\subsection{Effect of vibration on learning}

Our second hypothesis was that the Experimental group would integrate vibration induced afferent information in a way that improved their sensitivity to joint position. Contrary to our second hypothesis, there was neither an interaction between group and time nor a main effect of group for either constant or absolute error. It is important to point out, that although vibration was not beneficial, there were no negative effects of vibration on learning either. With training, subjects seem to be better at distinguishing their joint position even when extraneous haptic information is present. Consequently, low frequency vibration may have been ignored because it was irrelevant.

A previous study reported benefits in reduction of error of an elbow joint positioning task when $15 \mathrm{~Hz}$ vibration was applied [4]. However, in that particular study, vibration was not applied directly to the musculo-tendon of the elbow joint [4], and this fact may well account for the differences between our results. The indirect nature of the stimulation may make it more similar to whole body vibration, which has been shown to induce more positive effects on neuromuscular adaptation and motor performance than direct musculo-tendon stimulation [9].

It is apparent that low frequency vibration was not beneficial for our healthy adult cohort. This may be because in an intact sensory motor system, continuous vibration sensory input is down weighted. According to the sensory reweighting hypothesis, various sensory inputs are reweighted depending on the relevance to the task [10]. For example, if provided both visual and haptic feedback during a postural sway task, when haptic feedback became less reliable, sensitivity to visual feedback was greater [10]. Other studies have also shown a reduction in postural responses to vibration over time suggesting down weighting of irrelevant sensory stimuli generated by musculo-tendon vibration $[11,12]$.

Additionally, some research suggests that vibration may only be beneficial for individuals with impaired sensory systems. In a previous study, individuals with neuropathy experienced a favorable effect of vibration on center of pressure displacement and velocity under cognitive loading while healthy persons did not benefit [13]. In a cohort of persons diagnosed with unilateral stroke, vibration applied to the affected leg was more effective than on the unaffected leg in a walking task [14]. In light of this evidence, it is reasonable to surmise that continuous, low frequency vibration is not beneficial in a healthy system, but may be useful when sensation is impaired. Alternatively, continuous vibration has been found to be a useful stimulus when it is indicative of necessary changes in performance. For example, one group has successfully utilized vibration as a stimulus when changes in its amplitude indicated to the individual that a change in precision force or motor unit activity was required to optimally complete the task $[5,6]$.

Alternatively, the sensation of vibration may have simply weakened after the initial exposure since the frequency was so low. There are three possible reasons for this. First, vibration frequencies less than $50 \mathrm{~Hz}$ are known to be harder to distinguish than those of higher frequencies [15]. Second, a study of electrocutaneous sensitivity indicated that perception of haptic information is inhibited during movement [16]. In agreement with this, our subjects confirmed that they adequately perceived the vibration prior to beginning the experiment, but later commented that the sensation tended to disappear during movement of the knee on later trials. This is supportive of the sensory reweighting hypothesis. Third, continuous stimulation may result in less favorable perception than intermittent stimulation due to sensory habituation [17]. However, since vibration was turned off between 
trials, and individual trials lasted only seconds, habituation is unlikely to be an influencing factor in this study. Therefore, the most plausible explanation remains that because the sensory overflow associated with $15 \mathrm{~Hz}$ vibration was not providing informational content and was therefore irrelevant to the task being performed, it was down weighted by the sensory system and effectively ignored.

\subsection{Effects of vibration on transfer}

Our third hypothesis was that practice with vibration of the right leg would lead to greater bilateral transfer of learning to the left leg within the Experimental group relative to the Control group. While both absolute error and constant error transfer indices indicated that positive bilateral transfer occurred with practice, there were no differences in either of these measures between groups. Together this fails to support our hypothesis that vibration would increase bilateral transfer of a proprioception task at the knee joint.

The purpose of this element of this study was specifically directed at potential benefits for persons with transtibial amputations. The intention was to predict whether training with the intact limb would result in greater perceptual benefits in the affected limb and better quality of control of their prosthesis. However, as stated earlier, it appears that vibration is not useful in an intact system, which indicates that practice with vibration on the intact limb would not result in any perceptual advantages that could transfer to control of the affected limb and prosthesis.

\subsection{Conclusions}

In conclusion, a $15 \mathrm{~Hz}$ frequency of vibration on the quadriceps tendon did not prove to be an effective means of enhancing learning or bilateral transfer of a knee joint positioning task over the benefit of practice alone. However, $15 \mathrm{hz}$ vibration was also not found to be detrimental to learning of a knee joint position sensing task. One limitation of this study is that variability within groups was large contributing to small effect sizes, $\mathrm{n}_{\mathrm{p}}^{2}=.16$ for the main effect of time on absolute error and $n^{2}{ }_{p}=.18$ for the interaction of time with leg on constant error.

Possible alternatives for future research may be the comparison of several different frequencies, which may result in identification of a frequency of vibration in the lower spectrum that is beneficial. Frequency of vibration has been shown to modulate the response to stimulation in the past [3]. Next, a direct comparison of local tendon stimulation and local indirect stimulation may provide further insight into the differences between the effects of these types of stimulation. Finally, a comparison between groups of individuals with intact and impaired sensation may aid in determining whether this is a factor in the integration of vibration induced afferent information.

In summary, practice resulted in improvement of error on a knee proprioception task regardless of whether vibration induced afferent information was available during practice. Since practice alone was enough to improve performance on the task, the vibration stimulus was most likely down weighted because it was irrelevant. Thus, low frequency vibration may only be beneficial if the vibration induced haptic input is meaningfully related to the task or in situations where sensory feedback is impaired.

\section{ACKNOWLEDGEMENTS}

Brett Siders for assistance with pilot data collection. Lauren Gulley for assistance with data collection.

\section{REFERENCES}

[1] G. M. Goodwin, D. I. Mccloskey, and P. B. C. Matthews, "Proprioceptive Illusions Induced by Vibration : Contribution by Muscle Spindles to Perception," Science, vol. 175, no. 4028, pp. 1382-1384, 1972.
[2] J. P. Roll, J. P. Vedel, and E. Ribot, "Alteration of proprioceptive messages induced by tendon vibration in man: a microneurographic study," Experimental Brain Research, vol. 76, pp. 213-222, 1989.

[3] P. J. Cordo, V. S. Gurfinkel, S. Brumagne, and C. Flores-Vieira, "Effect of slow, small movement on the vibration-evoked kinesthetic illusion," Experimental Brain Research., vol. 167, no. 3, pp. 324-34, Dec. 2005.

[4] B. L. Tripp, D. Faust, and P. Jacobs, "Elbow joint position sense after neuromuscular training with handheld vibration," Journal of Athletic Training, vol. 44, no. 6, pp. 617-23, 2009.

[5] C. E. Stepp, C. Chang, M. Malhotra, \& Y. Matsuoka. "Vibrotactile feedback aids EMG control of object manipulation," Conference proceedings : ... Annual International Conference of the IEEE Engineering in Medicine and Biology Society, 2011: 1061-4, 2011.

[6] C. E. Stepp, Q. An \& Y. Matsuoka. Repeated training with augmented vibrotactile feedback increases object manipulation performance. PloS one, 7:e32743, 2012.

[7] C. J. Winstein and R. a. Schmidt, "Reduced frequency of knowledge of results enhances motor skill learning," Journal of Experimental Psychology: Learning, Memory, and Cognition, vol. 16, no. 4, pp. 677-691, 1990.

[8] D. S. Reisman, R. Wityk, K. Silver, and A. J. Bastian, "Split-belt treadmill adaptation transfers to overground walking in persons poststroke," Neurorehabilitation and neural repair, vol. 23, no. 7, pp. 735-44, Sep. 2009.

[9] C. Cardinale, M. \& Bosco, "The use of vibration as an exercise intervention," Exerc. Sci. Rev., vol. 31, no. 1, pp. 3-7, 2003.

[10] K. S. Oie, T. Kiemel, and J. J. Jeka, "Multisensory fusion: simultaneous re-weighting of vision and touch for the control of human posture," Brain research. Cognitive brain research, vol. 14, no. 1, pp. 164-76, Jun. 2002.

[11] P. A. Fransson, F. Tjernstron, A. Hafstrom, M. Magnusson, and R. Johansson, "Analysis of short- and long-term effects of adaptation in human postural control," Biological Cybernetics, vol. 86, pp. 355$365,2002$.

[12] P. A. Fransson, R. Johansson, F. Tjernstrom, and M. Magnusson, "Adaptation to vibratory perturbations in postural control," IEEE Engineering in Medicine and Biology Magazine, vol. 22, no. April, pp. 53-57, 2003.

[13] J. M. Hijmans, J. H. B. Geertzen, W. Zijlstra, A. L. Hof, and K. Postema, "Effects of vibrating insoles on standing balance in diabetic neuropathy," Journal of Rehabilitation Research \& Development, vol. 45, pp. 1441-1450, 2008.

[14] S.-I. Lin, L.-J. Hsu, and H.-C. Wang, "Effects of Ankle Proprioceptive Interference on Locomotion After Stroke.," Archives of physical medicine and rehabilitation, vol. 93, pp. 1027-1033, 2012.

[15] E. C. Wentink, A. Mulder, J. S. Rietman, and P. H. Veltink, "Vibrotactile stimulation of the upper leg: effects of location, stimulation method and habituation," Annual International Conference of the IEEE Engineering in Medicine and Biology Society, 1668-1671, Sept. 2011.

[16] R. W. Angel and R. C. Malenka, "Velocity-dependent suppression of cutaneous sensitivity during movement," Experimental Neurology, vol. 77, pp. 266-274, 1982.

[17] D. G. Buma, J. R. Buitenweg, and P. H. Veltink, "Intermittent stimulation delays adaptation to electrocutaneous sensory feedback.," IEEE Transactions on Neural Systems and Rehabilitation Engineering, vol. 15, 435-41, Sep. 2007. 